\title{
The Substitution Effect of Boron on Reentrant Behavior of Rapidly Solidified FeMnZr Alloys
}

\author{
Y. M. Moon ${ }^{1}$, K. S. Kinm ${ }^{1}$, S. C. Yü ${ }^{1}$ and V. Srinivas ${ }^{2}$ \\ ${ }^{I}$ Department of Physics, Chungbuk National University, Cheongju 361-763, Korea \\ ${ }^{2}$ Department of Physics, Indian Institute of Technology, Kharagpur-721 302, India
}

(Received 10 December 2004)

\begin{abstract}
The magnetization properties have been measured for amorphous $\mathrm{Fe}_{82} \mathrm{Mn}_{8-\mathrm{x}} \mathrm{B}_{\mathrm{x}} \mathrm{Zr}_{10}(\mathrm{x}=\mathbf{0 - 8})$ alloys. The temperature dependence of magnetization for these alloys shows the existence of antiferromagnetic couplings between Fe atoms in low fields at low temperatures. The magnetic parameters, obtained from the magnetization behavior are consistent with the presence of mixed magnetic state. The Curie temperature and magnetic moment increased with an increase of the concentration of $B$ and spin glass like transition observed at low temperature decreases and finally vanishes at $x=8$ at\%. Our result suggests that the substitution of $B$ for Mn seems to cause an increase of magnetic order.
\end{abstract}

Key words : reentrant behavior, antiferromagnetic coupling, spin fluctuation scattering

\section{Introduction}

Rapidly solidified Fe-Zr-B alloys with high Fe content exhibt excellent soft magnetic properties while amorphous $\mathrm{Fe}-\mathrm{Zr}-\mathrm{Mn}$ shows disordered magnetic phase with strong competing exchange interaction $[1,2]$. Recently it has been reported that the destruction of ferromagnetism results in enhancement of spin dependent electrical transport $[3,4]$. Intense efforts have carried out to understand the nature of the magnetic phase diagram from various magnetic measurements in amorphous Fe-Zr-Mn alloys $[5,6]$. The detailed analysis of magnetization data indicated that Mn substitution leads to enhancement of spin fluctuations while application of external magnetic field suppresses the same. Although the study of spin glass behavior in quenched random site Heisenberg ferromagnets has been actively pursued for more than a decade, there are still uncertainties in our basic understanding of this phenomenon. Theoretical spin fluctuation model seems to provide more realistic information on temperature variation of magnetization. For this reason, it would be interested to understand the effects of B substitutions in amorphous Fe-Zr-Mn alloys. In the present investigation, we report the synthesis and magnetic pro-

*Corresponding author: Tel: +82-43-261-2268;

e-mail: ymmoon@chungbuk.ac.kr perties of rapidly solidified $\mathrm{Fe}_{82} \mathrm{Mn}_{8} \mathrm{Zr}_{10}$ alloys substituted with $\mathrm{B}$.

\section{Experimental}

Ribbons of $\mathrm{Fe}_{82} \mathrm{Mn}_{8-\mathrm{x}} \mathrm{B}_{\mathrm{x}} \mathrm{Zr}_{10}(\mathrm{x}=0,2,4,6$ and 8) were prepared by single roller melt spinning under Argon atmosphere. The amorphous nature of the samples was confirmed by $\mathrm{X}$-ray diffraction using $\mathrm{Cu}-K \alpha$ radiation. Magnetic measurements were performed using a superconducting quantum interference device (SQUID) and vibrating sample magnetometer (VSM) in the field of 10 Oe or $10 \mathrm{kOe}$ and at the temperatures from $5 \mathrm{~K}$ up to 400 $\mathrm{K}$. Low field ac susceptibility data have been taken using conventional mutual induction technique to identify the magnetic phase transition temperatures.

\section{Results and Discussions}

The presence of lower temperature transition has been confirmed from dc and ac magnetic measurements. The typical plot of magnetization behavior as a function of temperature for $\mathrm{x}=0$ and $\mathrm{x}=6$ for $\mathrm{Fe}_{82} \mathrm{Mn}_{8-\mathrm{x}} \mathrm{B}_{\mathrm{x}} \mathrm{Zr}_{10}$ samples are shown in Figure 1. Our preliminary magnetic measurements on these samples indicate that $\mathrm{x}=0$ sample exhibits reentrant behavior with Curie temperature $\left(T_{\mathrm{c}}\right)=$ $195 \mathrm{~K}$ and spin freezing temperature $\left(T_{\mathrm{sg}}\right)=54 \mathrm{~K}$ as 


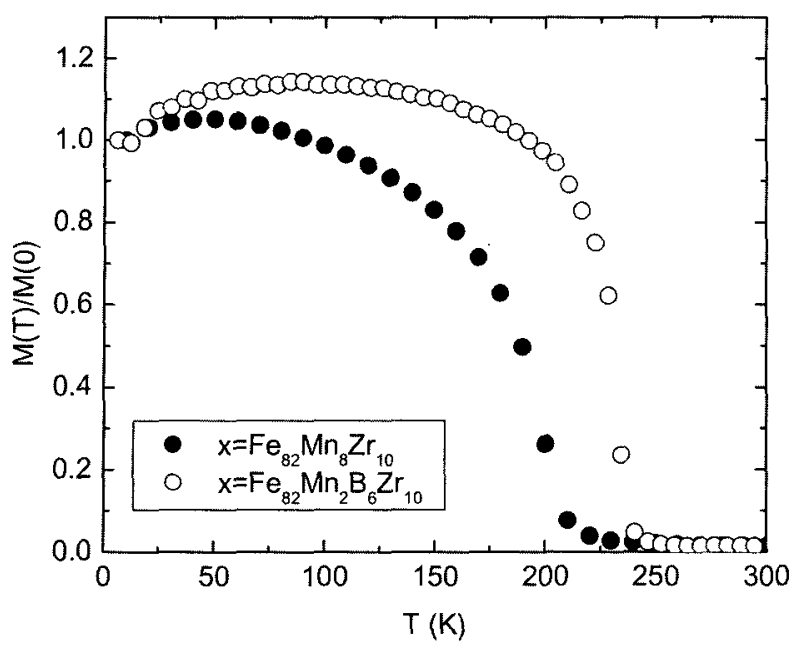

Fig. 1. Temperature dependence of magnetization at field of 10 kOe for amorphous $\mathrm{Fe}_{82} \mathrm{Mn}_{8} \mathrm{Zr}_{10}$ and $\mathrm{Fe}_{82} \mathrm{Mn}_{2} \mathrm{~B}_{6} \mathrm{Zr}_{10}$ alloys.

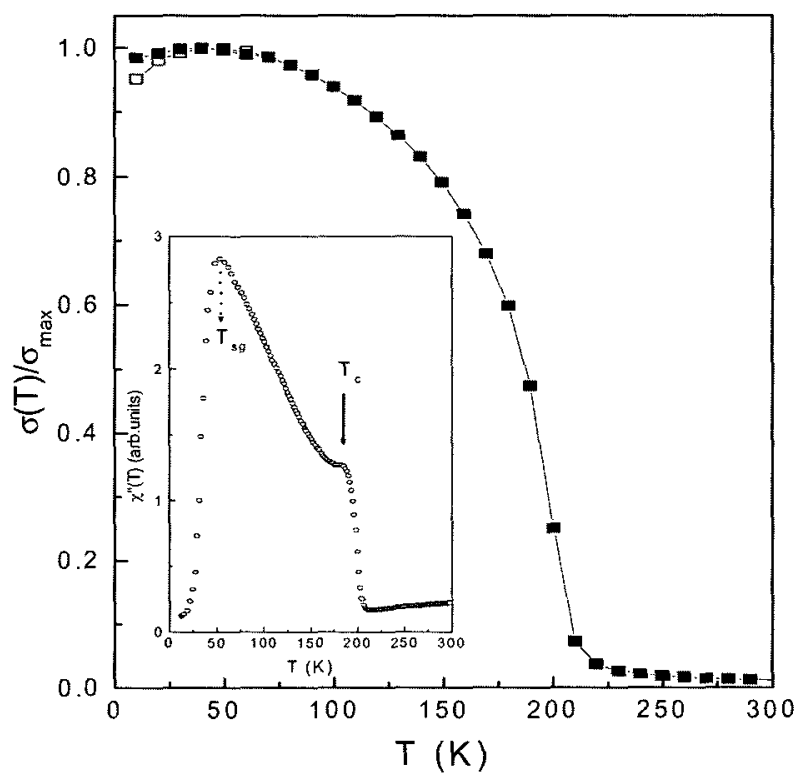

Fig. 2. The normalized value of field cooled and zero-field cooled magnetization curves with respect to maximum value in the curve, $\sigma_{\max }$, is plotted as a function of temperature for amorphous $\mathrm{Fe}_{82} \mathrm{Mn}_{8} \mathrm{Zr}_{10}$ alloy. Inset: The secondary component of ac susceptibility for amorphous $\mathrm{Fe}_{82} \mathrm{Mn}_{8} \mathrm{Zr}_{10}$ alloys.

shown in Figure 2 [7]. With an increase of the concentration of $\mathrm{Mn}$ for $\mathrm{Fe}_{90-x} \mathrm{Mn}_{x} \mathrm{Zr}_{10}$ systems, the Curie temperature decreases almost linearly and then the reentrant behavior is observed in all the samples. Figure 3 and 4 represent the spin freezing temperature, $T_{\mathrm{sg}}$ obtained from the field cooled (FC) and zero-field cooled (ZFC) magnetization curves as a function of temperature at 10 Oe field and Curie temperature as a function of $\mathrm{B}$ content for the amorphous $\mathrm{Fe}_{82} \mathrm{Mn}_{8-\mathrm{x}} \mathrm{B}_{\mathrm{x}} \mathrm{Zr}_{10}(\mathrm{x}=0 \sim 8)$ alloys,

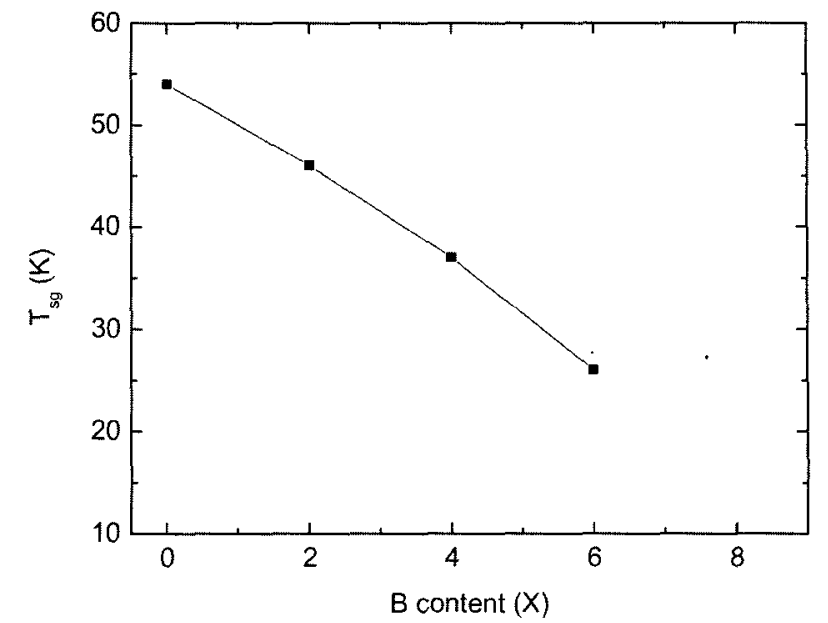

Fig. 3. Spin freezing temperature in field of $10 \mathrm{Oe}$ as a function of $\mathrm{B}$ concentration for amorphous $\mathrm{Fe}_{82} \mathrm{Mn}_{8-\mathrm{x}} \mathrm{B}_{\mathrm{x}} \mathrm{Zr}_{10}(\mathrm{x}=0$ 8) alloys.

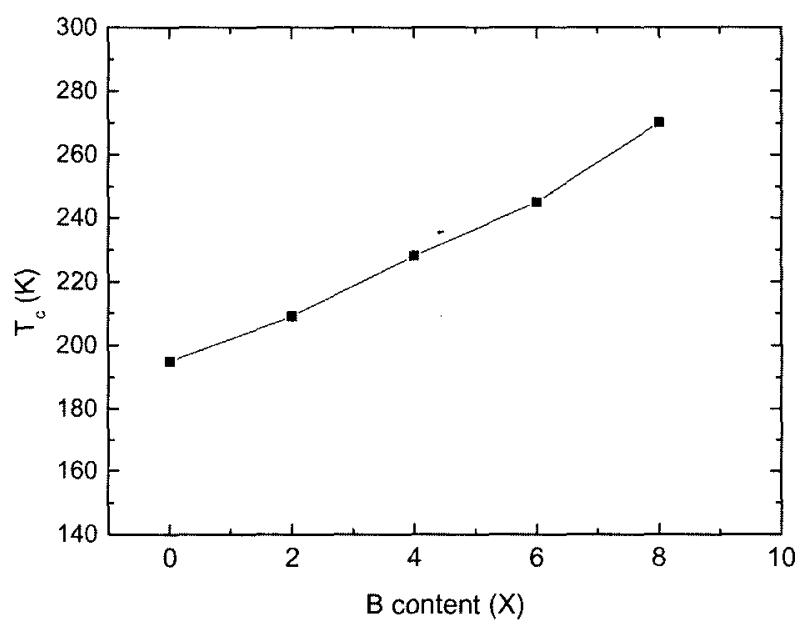

Fig. 4. Curie temperature as a function of $B$ concentration for amorphous $\mathrm{Fe}_{82} \mathrm{Mn}_{8-\mathrm{x}} \mathrm{B}_{\mathrm{x}} \mathrm{Zr}_{10}(\mathrm{x}=0-8)$ alloys.

respectively. The low field thermo-magnetization data show a clear double transition behavior below room temperature in all samples. It is important to know whether the spin glass like behavior at low temperature originates from the competing exchange interactions or random magnetic anisotropy. As boron increases, the spin glass like transition observed at low temperature decreases from $54(\mathrm{x}=0)$ to $26 \mathrm{~K}(\mathrm{x}=6)$ and finally vanishes at $\mathrm{x}=$ 8 at \%. The Curie temperature increases with increasing of B concentration. Thus the increase of $T_{\mathrm{c}}$ with the substitution of $\mathrm{B}$ with $\mathrm{Mn}$ may be explained from the possible increase of the magnetic moment of $\mathrm{Fe}$ and the possible increase of the number of ferromagnetic couplings between $\mathrm{Fe}$ atoms [3]. The non-saturation behavior of magnetization curves in applied field of more than 60 


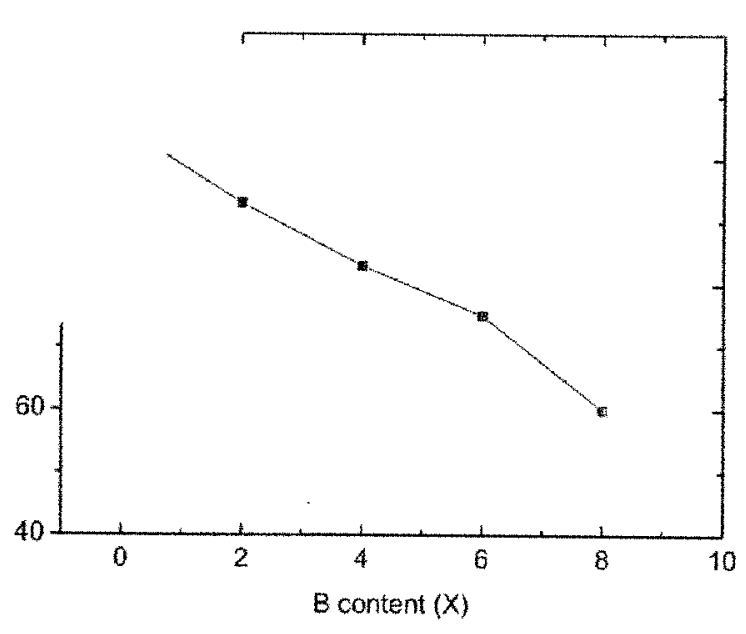

Fig. 5. Coercivity as a function of $B$ concentration for amorphous $\mathrm{Fe}_{82} \mathrm{Mn}_{8-\mathrm{x}} \mathrm{B}_{\mathrm{x}} \mathrm{Zr}_{10}(\mathrm{x}=0-8)$ alloys.

kOe is observed for $\mathrm{Fe}_{82} \mathrm{Mn}_{8} \mathrm{Zr}_{10}$ alloy, while boron substitution results in reduction of high field susceptibility, This suggests a possible presence of competing exchange interaction. In disordered $\mathrm{Fe}$-rich $\mathrm{Fe}-\mathrm{Zr}$ alloys it is known that the magnetization can not be saturated by weak applied magnetic fields due to the competition between ferromagnetic and antiferromagnetic interactions.

From the analysis by approaching the saturation magnetization, $M_{s}$ at low temperature, the spin moment of Fe and exchange stiffness, $A$, can be extracted. It is well known that $A$ can drive the following relation [8],

$$
A=\left(C S_{F e} k_{B} T_{c}\right) / 4\left(S_{F e}+1\right) r_{F e-F e} \text {, }
$$

where $C$ is the concentration of Fe in atomic percent, $S_{F_{e}}$ the spin moment of $\mathrm{Fe}$ and $r_{F e-F e}$ is the nearest neighbor distance. The magnetic moment of the alloy arises from the total contribution of the moment of $\mathrm{Fe}\left(\mu_{F e}\right)$, which is aligned ferromagnetically. We can calculate the $S_{F e}$, from the relation of $M\left(\mu_{B}\right)=2 \chi_{F e} S_{F e} \mu_{B}$, where $\chi_{F e}$ is the concentration of $\mathrm{Fe}$. The values of $A$ are found to be 16.8, $17.6,19.9,21.3$ and $22.2\left(10^{-8} \mathrm{erg} / \mathrm{cm}\right)$ for $\mathrm{x}=0,2,4,6$ and 8 , respectively. And also the $S_{F e}$ was found to be increases with $\mathrm{B}$ concentration. This result can be explained in terms of a increase of ferromagnetic couplings with boron content. Figure 5 shows the coercivity, $H_{\varepsilon}$ for the amorphous $\mathrm{Fe}_{82} \mathrm{Mn}_{8-\mathrm{x}} \mathrm{B}_{\mathrm{x}} \mathrm{Zr}_{10}(\mathrm{x}=0-8)$ ailoys. The coercivity values measured at $5 \mathrm{~K}$ decrease with increasing boron content. These observations indicate that the magnetic frustration decreases as the boron concentration increased and results in development of better soft magnetic properties.

In conclusion, $\mathrm{B}$ substitution to $\mathrm{Fe}_{82} \mathrm{Mn}_{8-\mathrm{x}} \mathrm{B}_{\mathrm{x}} \mathrm{Zr}_{10}$ alloys shows a monotonous increases in Curie temperature and spin glass like transition observed at low temperature decreases and finally vanishes at $x=8$ at $\%$. This result can be explained in terms of a increases of ferromagnetic coulplings with increasing of $B$ concentration.

\section{Acknowledgement}

This work was supported by the Chungbuk National University Grant in 2004.

\section{References}

[1] G. K. Nicolaides, G. C. Hadjipanayis, and K. V. Rao, Phys. Rev. 48, 12759 (1993).

[2] P. D. Babu and S. N. Kaul, Condens. Matter 9, 7189 (1997).

[3] A. Perumal, V. Srinivas, K. S. Kim, S. C. Yu, V. V. Rao, and R. A. Dunlap, Phys. Rev. 65, 064428 (2002).

[4] K. Suzuki, J. W. Cochrane, K. Aoki, and J. M. Cadogan, J. Magn. Magn. Mater. 242-245, 273 (2002).

[5] J. M. D. Coey, D. H. Ryan, and R. Buder, Phys, Rev. Lett. 58, 385 (1987).

[6] D. Dahlberg, K. V. Rao, and K. Fukamichi, J. Appl. Phys. 53, $2310(1982)$.

[7] K. S. Kim, S. C. Yu, A. Perumal, and V. Srinivas, J. Magn. Magn. Mater 254-255, 100 (2003).

[8] R. Hasegawa, I. Appl. Phys. 45, 3109 (1974). 